\title{
Screening tools for child sexual abuse
}

\author{
Vincent J. Palusci, ${ }^{1}$ John V. Palusci ${ }^{2}$
}

\begin{abstract}
The medical diagnosis of child sexual abuse can be problematic; any opportunity to simplify, strengthen, or streamline the assessment process is desirable. Screening has been defined as the presumptive identification of unrecognized disease or defect by the application of tests, examinations or other procedures which can be carried out rapidly with the goal of sorting out apparently well persons who probably have a disease from those who do not. ${ }^{1}$ Screening for child sexual abuse
\end{abstract} is potentially helpful by either identifying victims in general pediatric populations where no concerns have previously arisen or by providing pediatricians with additional information to direct their diagnostic process for children presenting with symptoms of potential sexual abuse.

A screening test is itself not intended to be diagnostic, and a national task force in the United States found insufficient evidence to recommend for or against routine screening of parents or guardians for family violence. ${ }^{2}$

Screening has been used for many conditions in medicine, and a multitude of instruments has become available for identifying child physical abuse, sexual abuse and neglect. ${ }^{3-6}$ Historically, the initial purpose of screening was to learn the distinguishing characteristics of sexually abused and non-abused children. Screening tests have been administered to almost anyone in contact with the child: parents, investigators, and children themselves. One potential indicator of sexual abuse is sexual behavior, which can be assessed via parent report using items such as those from the Child Behavior Checklist and the Child Sexual Behavior Inventory. ${ }^{7}$ These inventories and their updated versions can probabilistically distinguish between the sexual behaviors of normative and sexually abused children, but are less reliable in children undergoing psychiatric or

1. MD, MS. Wayne State University, Detroit, MI, USA.

2. University of Pennsylvania, Philadelphia, PA, USA.

Suggested citation: Palusci VJ, Palusci JV. Screening tools for child sexual abuse. J Pediatr (Rio J). 2006;82:409-10.

doi: $10.2223 / J P E D .1572$ psychological treatment. Physical examination findings can be helpful, but when they are normal, they neither "rule out" nor support allegations of sexual abuse, 8 since not all sexual abuse leaves identifiable physical evidence. An assessment tool using behavior, disclosure and physical findings has been used by many medical professionals in the United States, but this tool has never been intended for screening in the general population.8 After medical assessment, final substantiation of abuse rests on the confessions of perpetrators and findings in the child welfare or legal systems, which make the final determination and initiate steps for the protection of the child. ${ }^{9}$

In this issue, Salvagni \& Wagner ${ }^{10}$ describe the development and implementation of a screening questionnaire for child sexual abuse that is administered to parents during a medical assessment of their child, aged 2-12 years. This 5 -item survey screens for behavioral symptoms using items previously studied in other validated but lengthier instruments. ${ }^{71}$ Beginning with 18 items, they narrowed their questionnaire down to five questions that together can reasonably distinguish their control set of non-abused children from those seen in a clinic for sexual violence. Scores reflecting positive answers to three or more questions significantly raised the probability of sexual abuse, with acceptable test characteristics. Depending on the environment of the screen (either in a normal clinic or one for victims of sexual violence), different scores would presumably set into motion other multidisciplinary assessments to confirm or "rule out" the diagnosis of sexual abuse and, if necessary, initiate legal protective measures.

Constructing a meaningful screening tool is difficult for the medical assessment of sexual abuse, and Salvagni \& Wagner are to be complimented for their parsimony. In isolation, various assessments such as word association, anatomical doll play, and other indirect psychological evaluations have not adequately discriminated between normative, physically abused and sexually abused children. ${ }^{12}$ Child sexual abuse 
takes place within a sociocultural context, and diagnostic decisions are greatly influenced by the estimated costs of a false diagnosis, with its risk of misclassifying children who are being sexually abused and subjecting them to further trauma against the risk of misclassifying children who are not being abused and subjecting them and their families unnecessarily to the stress of intervention. ${ }^{12}$ Sexually abused children live in significantly more aversive and stressful circumstances than non-abused children, with poorer families, less educated parent(s), and experience more stressful events over and above their sexual abuse, further confounding any assessment. ${ }^{7}$ While behavioral symptoms and disclosure are important in medical treatment and child protective services investigations, positive physical findings, though uncommon, are highly associated with guilty verdicts. ${ }^{9}$ A review of several existing assessment and screening tools for trauma in children and adolescents concluded that no one measure is conditioned to every situation and that new instruments are needed to fill gaps in current assessment procedures. ${ }^{6}$ Salvagni \& Wagner's instrument begins to address these issues in that it combines items from standardized instruments with physical examination findings.

It is interesting to note what items were not included in Salvagni \& Wagner's final instrument. While abnormal curiosity about the genitals, fear of being left with a given person, sudden emotional or behavioral changes, abandonment of previous play habits, and anogenital injuries were included, problems with sleep, somatic complaints, easy crying, aggressive sexualized play, excessive masturbation, fear of being left alone, problems in school, sexual knowledge, bedwetting, and parental history of sexual victimization were not. Earlier studies have showed a correspondence between many of these behavior problems and other stressful events with or without sexual abuse, and many of the items left out are helpful in determining which child could benefit from counseling and treatment irrespective of their predictive value of sexual abuse. 5,7,9

Salvagni \& Wagner's study does suffer from some limitations. Their sample size is relatively small, with 192 children from one area. This will affect its generalizability to other populations of children. As with all child abuse studies, the lack of a "gold standard" in making sure the cases are properly identified and the controls are "free" from abuse is problematic. While this is taken into consideration with the authors' assumption of $5 \%$ prevalence of sex abuse in the general clinic and $40 \%$ in the referral center, additional evaluation in the control group and longer follow-up could reduce potential misclassification. Lastly, while it is laudable not to require children to undergo additional interviews and medical procedures for research purposes alone, the authors' use of prior disclosures and examination findings affects the reliability of some of the answers to their instrument.

Despite these shortcomings, it is our opinion that pediatricians can use Salvagni \& Wagner's questionnaire to identify children with an increased risk in the general population and to identify those at greatest risk among those referred for possible sexual abuse. For children in the general pediatric clinic, a positive screen should initiate additional pediatric and psychosocial assessment and treatment for behavior problems. In the referral setting, close examination by child welfare and other investigators will be needed, and identifying children at highest risk can help medical professionals make appropriate treatment and referral recommendations, particularly when resources are scarce. From the medical perspective, this questionnaire is not intended as "proof" of abuse; only as a prompt to begin gathering additional information surrounding observed concerns. Also, a negative screen should not rule out the possibility of abuse if other compelling evidence exists. This is precisely how a screen should be implemented: as a measure used to initiate further, more discriminatory assessments to confirm child sexual abuse, and not as a "make-or-break" indicator. Salvagni \& Wagner are to be commended for developing a simple but elegant instrument, and we look forward to furthering its study in larger, more diverse populations with long-term follow-up to show the positive outcomes associated with earlier identification and treatment of child sexual abuse.

\section{References}

1. Last JM, editor. A dictionary of epidemiology. 3rd ed. New York: Oxford University Press; 1995.

2. U.S. Preventative Services Task Force. Screening for family and intimate partner violence: recommendation statement. Ann Fam Med. 2004;2:156-60.

3. Lounds JJ, Borkowski JG, Whitman TL; The Centers for the Prevention of Child Neglect. Reliability and validity of the motherchild neglect scale. Child Maltreat. 2004;9:371-81.

4. Chang DC, Knight VM, Ziegfeld S, Haider A, Paidas C. The multiinstitutional validation of the new screening index for physical child abuse. J Pediatr Surg. 2005;40:114-9.

5. Hulme PA. Retrospective measurement of childhood sexual abuse: a review of instruments. Child Maltreat. 2004;9:201-17.

6. Strand VC, Sarmiento TL, Pasquale LE. Assessment and screening tools for trauma in children and adolescents: a review. Trauma Violence Abuse. 2005;6:55-78.

7. Friedrich WN, Fisher JL, Dittner CA, Acton R, Berliner L, Butler J, et al. Child Sexual Behavior Inventory: normative, psychiatric, and sexual abuse comparisons. Child Maltreat. 2001;6:37-49.

8. Adams JA. Evolution of a classification scale: medical evaluation of suspected child sexual abuse. Child Maltreat. 2001;6:31-6.

9. Palusci VJ, Cox EO, Cyrus TA, Heartwell SW, Vandervort FE, Pott ES. Medical Assessment and legal outcome in child sexual abuse. Arch Pediatr Adolesc Med. 1999;153:388-92.

10. Salvagni EP, Wagner MB. Development of a questionnaire for the assessment of sexual abuse in children and estimation of its discriminant validity: a case-control study. J Pediatr (Rio J). 2006;6:431-6.

11. Wells R, McCann J, Adams J, Voris J, Dahl B. A validational study of the Structured Interview of Symptoms Associated with Sexual Abuse (SASA) using three samples of sexually abused, allegedly abused, and nonabused boys. Child Abuse Negl. 1997;21:1159-67.

12. Babiker $G$, Herbert $M$. The role of psychological instruments in the assessment of sexual abuse. Child Abuse Negl. 1996;5: 239-51. 